\title{
Reduced levels of N'-methyl-2-pyridone-5-carboxamide and lysophosphatidylcholine 16:0 in the serum of patients with intrahepatic cholangiocarcinoma, and the correlation with recurrence-free survival
}

\author{
Kyung-Hee Kim¹,2, Jungnam Joo ${ }^{3}$, Boram Park ${ }^{3}$, Sang-Jae Park, Woo Jin Lee ${ }^{4}$, \\ Sung-Sik Han ${ }^{4}$, Tae Hyun Kim ${ }^{4}$, Eun Kyung Hong ${ }^{4}$, Sang Myung Woo ${ }^{1,4}$ and Byong \\ Chul Yoo ${ }^{1}$ \\ ${ }^{1}$ Biomarker Branch, Research Institute, National Cancer Center, Goyang 10408, Republic of Korea \\ ${ }^{2}$ Omics Core Laboratory, Research Institute, National Cancer Center, Goyang 10408, Republic of Korea \\ ${ }^{3}$ Biometrics Research Branch, Research Institute, National Cancer Center, Goyang 10408, Republic of Korea \\ ${ }^{4}$ Center for Liver Cancer, Hospital, National Cancer Center, Goyang 10408, Republic of Korea \\ Correspondence to: Sang Myung Woo, email: wsm@ncc.re.kr \\ Byong Chul Yoo, email: yoo_akh@ncc.re.kr
}

Keywords: metabolic biomarkers; intrahepatic cholangiocarcinoma

Received: August 30, $2017 \quad$ Accepted: October 25, 2017

Published: November 22, 2017

Copyright: Kim et al. This is an open-access article distributed under the terms of the Creative Commons Attribution License 3.0 (CC BY 3.0), which permits unrestricted use, distribution, and reproduction in any medium, provided the original author and source are credited.

\section{ABSTRACT}

We searched for metabolic biomarkers that may predict the prognosis of patients with intrahepatic cholangiocarcinoma (IHCC). To this end, a total of 237 serum samples were obtained from IHCC patients $(n=87)$ and healthy controls $(n=150)$, and serum metabolites were analyzed by liquid chromatography-tandem mass spectrometry (LC-MS/MS). Two stratified algorithms were used to select the metabolites, the levels of which predicted the prognosis of IHCC patients. We performed MS/MS and multiple-reaction-monitoring MS analyses to identify and quantify the selected metabolites. Continuous biomarker levels were dichotomized based on cutoffs that maximized between-group differences in recurrence-free survival (RFS) in terms of the log-rank test statistic. These RFS differences were analyzed using the log-rank test, and survival curves were drawn with the aid of the Kaplan-Meier method. Six metabolites (L-glutamine, lysophosphatidylcholine [LPC] 16:0, LPC 18:0, N'-methyl-2-pyridone-5-carboxamide [2PY], fibrinopeptide A [FPA] and uric acid) were identified as candidate metabolic biomarkers for predicting the prognosis of IHCC patients. Of these metabolites, levels of L-glutamine, uric acid, LPC 16:0, and LPC 18:0 were significantly lower in the serum from IHCC patients, whereas levels of 2PY and FPA were significantly higher $(p<0.01) .2$ PY and LPC 16:0 showed significantly better RFS at low level than high level (2PY, median RFS: 15.16 months vs. 5.90 months, $p=0.037$; LPC 16:0, median RFS: 15.62 months vs. 9.83 months, $p=0.035$ ). The findings of this study suggest that 2PY and LPC 16:0 identified by metabolome-based approaches may be useful biomarkers for IHCC patients.

\section{INTRODUCTION}

The biliary tract and drainage system include the intra- and extra-hepatic bile ducts and gallbladder. "Cholangiocarcinoma" refers to a tumor arising from the bile duct epithelium. Intrahepatic cholangiocarcinoma
(IHCC) is the second most common primary hepatobiliary cancer (after hepatocellular carcinoma). IHCCs are rare, difficult to diagnose, and have a poor prognosis [1]. Cholangiocarcinoma is generally rare in Western countries [2], but is more common in Korea, with about 3,500 cases diagnosed annually [3]. In 
addition, global mortality attributed to IHCC has recently increased [4].

IHCC diagnosis is complex, featuring a combination of appropriate clinical suspicion, imaging, endoscopy, and cytopathological examination. However, the fact that tumors are often detected in the late stages coupled with the poor prognosis of this disease, render it essential to identify biomarkers that allow early diagnosis. Carbohydrate antigen 19-9 (CA19-9), the most commonly used serum marker in clinical practice, is a sialylated Lewis blood group antigen than can be targeted by monoclonal antibodies. It was described in 1979 as a tumor-associated antigen in a colorectal cancer cell line [5]. CA19-9 measurements exhibit wide variation in sensitivity (50-90\%) and specificity (54-98\%), often being elevated in patients with benign biliary disease and/or cholangitis; the levels fall after relief of any biliary obstruction or sepsis $[6,7]$. Despite widespread use of serum CA19-9 levels in IHCC patients, their prognostic role is less clear. Cytokeratins (CKs) are intermediate filaments that are essential for cytoskeleton formation in epithelial cells. Twenty different CK polypeptides have been identified, and CK-19 is associated with cholangiocarcinoma. It appears that CK-19 is more specific for IHCC than for other tumor types, but its sensitivity for tumor diagnosis is low [8].

Recently, we analyzed metabolites that were detected as low-mass ions by mass spectrometry (MS), and reported the metabolic profiles of biofluids (including urine and serum) of cancer patients. The data were useful for the early cancer diagnosis and prediction of prognosis [9-15]. Here, we described the MS-based metabolic profile of serum from IHCC patients, and explored the clinical significance of the upregulation of N'-methyl-2pyridone-5-carboxamide levels in these patients.

\section{RESULTS}

\section{Patient characteristics}

The baseline characteristics of all of the IHCC patients are shown in Table 1. The median age (range) of the patients was 60 years (31-91 years), most of whom were males $(70.1 \%)$. Of the 87 patients, $43 \%$ had $\mathrm{T} 1$ disease and $37 \%$ had lymph node (LN) metastasis. In addition, 41 (51\%) had high CA19-9 levels (>37 U/mL) and 18 (21\%) had chronic hepatitis B; Common bile duct stones were found in 3 patients $(3.5 \%)$, and gallbladder stones were exhibited in 7 patients $(8.1 \%)$. Serum CA 19-9 level was not associated with recurrence-free survival (RFS), even at a very high cut-off of 1,000 U/mL (Table 2).

\section{Identification of six metabolites differentially expressed in the serum of IHCC patients}

Six metabolic ions that were differentially expressed in IHCC patients were identified with the aid of MarkerView software as described in the Materials and Methods section. The ion of $153.0659 \mathrm{~m} / \mathrm{z}$ was attributable to one of two possible compounds: N'methyl-2-pyridone-5-carboxamide (2PY) (HMDB04193) or N'-methyl-4-pyridone-5-carboxamide (4PY) (HMDB04194) (Figure 1A, 1B). Because the chemical structures of 2PY and 4PY are very similar (Figure 1C), the retention times (RTs) of both compounds were almost similar ( 4.41 min) (Figure 1B). However, only 2PY exhibited a tandem mass spectrometry (MS/MS) pattern identical to that of the ion of $153.0659 \mathrm{~m} / \mathrm{z}$ (Figure 1D). Similarly, two metabolites, L-glutamine (HMDB00641) and ureidoisobutyric acid (HMDB02031), were candidates for the origin of the metabolic ion of 147.0764 $\mathrm{m} / \mathrm{z}$ with an RT of $1.44 \mathrm{~min}$, but MS/MS analysis revealed that the material was in fact L-glutamine (Figure 2). Lysophosphatidylcholine (LPC) 18:0 was the only candidate for the origin of the metabolic ion of 524.3711 $\mathrm{m} / \mathrm{z}$ with an RT of $16.32 \mathrm{~min}$, and the MS/MS patterns of the two molecules were identical (Figure 3). The metabolic ion of (negative) $167.0211 \mathrm{~m} / \mathrm{z}$ with an RT of $2.48 \mathrm{~min}$ was shown to have originated from uric acid (HMDB00289) (Figure 4). Two other metabolic ions (of $496.3398 \mathrm{~m} / \mathrm{z}$ with an RT of $14.977 \mathrm{~min}$ and 768.8499 $\mathrm{m} / \mathrm{z}$ with an RT $7.544 \mathrm{~min}$ ) had been identified as LPC 16:0 [12] and fibrinopeptide A [9], respectively, in our previous studies.

\section{Quantification of the six differentially expressed metabolites in the serum from IHCC patients}

The six metabolites described above, as revealed by MS/MS, were quantified in serum from 87 IHCC patients and 150 healthy controls using stable isotope-labeled compounds and internal standards as described in the Materials and Methods section. Of the six metabolites, 2PY and FPA were elevated by ca. 2- and 2.5-fold in serum from IHCC patients $(2 \mathrm{PY}, 529.67 \pm 277.97 \mathrm{pg}$ / $\mu \mathrm{L}$; FPA, $158.86 \pm 82.07 \mathrm{pg} / \mu \mathrm{L}$ ) compared to the controls (2PY, $257.21 \pm 122.74 \mathrm{pg} / \mu \mathrm{L}$; FPA, $58.57 \pm 42.22 \mathrm{pg} /$ $\mu \mathrm{L}$ ) (both $p<0.01$ ) (Figure 5). By contrast, L-glutamine, uric acid, LPC 16:0, and LPC 18:0 levels were reduced in the serum from IHCC patients (Figure 5). Although L-glutamine and uric acid levels significantly decreased, the decrease did not extend to fold changes (L-glutamine, IHCC $68.32 \pm 13.63 \mathrm{ng} / \mu \mathrm{L}$ vs. control $77.95 \pm 11.90 \mathrm{ng} /$ $\mu \mathrm{L}$; uric acid, IHCC $29.45 \pm 8.78 \mathrm{ng} / \mu \mathrm{L}$ vs. control 36.40 $\pm 9.88 \mathrm{ng} / \mu \mathrm{L}$ ) (both $p<0.01$ ) (Figure 5). LPC 16:0 and LPC 18:0 were quantified by measuring their mass peak areas; the levels of both compounds were significantly reduced in the serum from IHCC patients (LPC 16:0, IHCC 20,818,908.35 $\pm 8,595,234.51$ arbitrary units [AUs] vs. control 34,163,977.98 $\pm 10,387,253.39 \mathrm{AU}$; LPC 18:0, IHCC 1,115,813.58 $\pm 814,923.58$ AU vs. control 2,972,574.94 $\pm 2,059,398.56 \mathrm{AU})$ (both $p<0.01$ ) (Figure 5). 
Table 1: Baseline characteristics of the total IHCC patients

\begin{tabular}{lll}
\hline Characteristics & Median (range) & Number (\%) \\
\hline Follow-up duration & Median (range) & $60(31-91)$ \\
Age & Male & $61(70.1)$ \\
Sex & 1 & $37(42.5)$ \\
T stage* & 2 & $21(24.1)$ \\
& 3 & $19(21.8)$ \\
& 4 & $10(11.5)$ \\
N stage1 & 1 & $23(36.5)$ \\
M stage & 1 & $5(5.8)$ \\
CA19-92 & $>37$ U/mL & $41(50.6)$ \\
Total bilirubin level & Median (range) & $0.6(0.2-12.2)$ \\
HBsAg & + & $18(20.7)$ \\
Anti-HBs & + & $47(54.0)$ \\
Anti-HBc3 & + & $68(84.0)$ \\
Anti-HCV4 & + & $3(3.5)$ \\
Liver cirrhosis & + & $13(14.9)$ \\
History of CBD stone & + & $3(3.5)$ \\
History of GB stone & + & $7(8.1)$ \\
\hline
\end{tabular}

"7th AJCC TNM staging system, missing data: $1 ; n=24,2 ; n=6,3 ; n=6 ; 4 ; n=1$

Common bile duct stone, CBD; gallbladder, GB.

Table 2: CA 19-9 level and RFS

\begin{tabular}{|c|c|c|c|c|c|}
\hline \multirow[b]{2}{*}{$\mathbf{U} / \mathbf{m L}$} & \multicolumn{3}{|c|}{ Frequency (\%) } & \multicolumn{2}{|c|}{ Cox (univariable) } \\
\hline & Total & No event & Event & HR (95\% CI) & $p$-value \\
\hline$\leq 22.9^{*}$ & 31 & $12(38.7)$ & $19(61.3)$ & 1 & \\
\hline$>22.9^{*}$ & 50 & $9(18.0)$ & $41(82.0)$ & $1.50(0.87-12.60)$ & 0.1454 \\
\hline$\leq 37$ & 40 & $12(30.0)$ & $28(70.0)$ & 1 & \\
\hline$>37$ & 41 & $9(22.0)$ & $32(78.1)$ & $1.30(0.78-2.16)$ & 0.3180 \\
\hline$\leq 100$ & 56 & $17(30.4)$ & $39(69.6)$ & 1 & \\
\hline$>100$ & 25 & $4(16.0)$ & $21(84.0)$ & $1.53(0.90-2.61)$ & 0.1178 \\
\hline$\leq 1000$ & 72 & $20(27.8)$ & $52(72.2)$ & 1 & \\
\hline$>1000$ & 9 & $1(11.1)$ & $8(88.9)$ & $2.04(0.96-4.33)$ & 0.0627 \\
\hline
\end{tabular}

*The cutoff was determined to maximize the differences between groups in terms of the log-rank test statistic.

\section{The 2PY and LPC 16:0 levels were associated} with RFS of IHCC patients

We determined the cutoff values of the six metabolites that maximized the RFS differences (Table 3). No value was significantly associated with $\mathrm{T}$ or $\mathrm{N}$ stage (Supplementary Table 1), but the RFS of those with low and high $2 \mathrm{PY}$ levels significantly differed (median; 15.16 months vs. 5.90 months; $p=0.037$ ) (Figure 6, Supplementary Table 2). 2PY and LPC 16:0 showed significantly better RFS at low level than high level (median; 15.62 months vs. 9.83 months; $p=0.035$ ). IHCC patients with high (compared to low) levels of L-glutamine experienced better overall survival (OS) (median; 48.20 months vs. 12.71 months; $p=0.0121$ ) (Figure 6).

\section{DISCUSSION}

We found that six metabolites (L-glutamine, LPC 16:0, LPC 18:0, 2PY, FPA, and uric acid) were candidate 
predictors of IHCC prognosis (Figures 1-4). Of these, the 2PY level was significantly increased in IHCC patients $(p<0.01)$ (Figure 5), and a high level significantly correlated with poor RFS (median; 15.16 months vs. 5.90 months, $p=0.037$ ) (Figure 6). Nicotinamide, 2PY, and 4PY are metabolites of the intracellular co-enzyme nicotinamide adenine dinucleotide (NAD), and potentially inhibit poly(ADP-ribose)polymerase (PARP)-1, a DNA repair enzyme [16]. NAD catabolism in mammalian cells occurs principally via a reaction catalyzed by PARP, releasing nicotinamide, which then is predominantly metabolized to 2PY [16, 17]. PARP is involved in DNA repair, the cell stress response, and regulation of apoptosis. Therefore, NAD metabolites including 2PY may accumulate under diseased conditions, accelerating DNA damage and causing the retention of catabolic products $[16,17]$.

NAD metabolites may be particularly harmful in children (who exhibit more DNA turnover than adults); NAD metabolites accumulate in the plasma of children with chronic renal failure [18]. In addition, the level of 2PY, an end product of NAD degradation, increases in the serum of chronic renal failure patients, and is associated with the deterioration of kidney function and toxicities including significant PARP-1 inhibition. 2PY was also elevated in the plasma of uremic patients [19]. Accumulation of $2 \mathrm{PY}$ in erythrocytes was positively correlated with the extent of renal failure [20]. Therefore,
A



C



HMDB04194

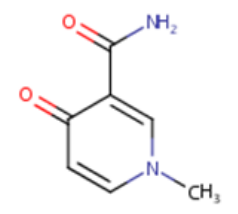

2PY may be a novel toxin that plays a significant role in the development of uremic toxemia, particularly by acting as a PARP inhibitor $[16,17,21]$. By contrast, one report found that $2 \mathrm{PY}$ protected endothelial cells from oxidative stress injury by inhibiting PARP and reducing NAD depletion. This maintained cellular energetics and high ATP levels [22].

Apart from the reported association with renal failure, the effects of $2 \mathrm{PY}$ on other diseases have received little attention. As liver cirrhosis progresses, the extent of nicotinamide methylation and urinary N-methylnicotinamide and 2-pyridone-5-carboxamide levels gradually rise [23]. Hypermethylation might protect against the toxic effects of intracellular nicotinamide attributable to cirrhosis-associated catabolism [23]. Further work is needed to explain why 2PY levels increase in the sera from IHCC patients; it may be a useful biomarker not only when screening for IHCC but also for predicting disease prognosis.

Unlike 2PY, the levels of LPC 16:0 and LPC 18:0 decreased in serum from IHCC patients $(p<0.01)$ (Figure 5), and low levels of LPC 16:0 (compared to high levels) significantly correlated with an improved RFS (median; 15.62 months vs. 9.83 months, $p=0.035$ ) (Figure 6). Lysophosphatidic acid (LPA; monoacylglycerol-3-phosphate) serves as a mitogen and a stimulator of motility in many cell types [24], acting in either an autocrine or paracrine fashion via $G$ protein-

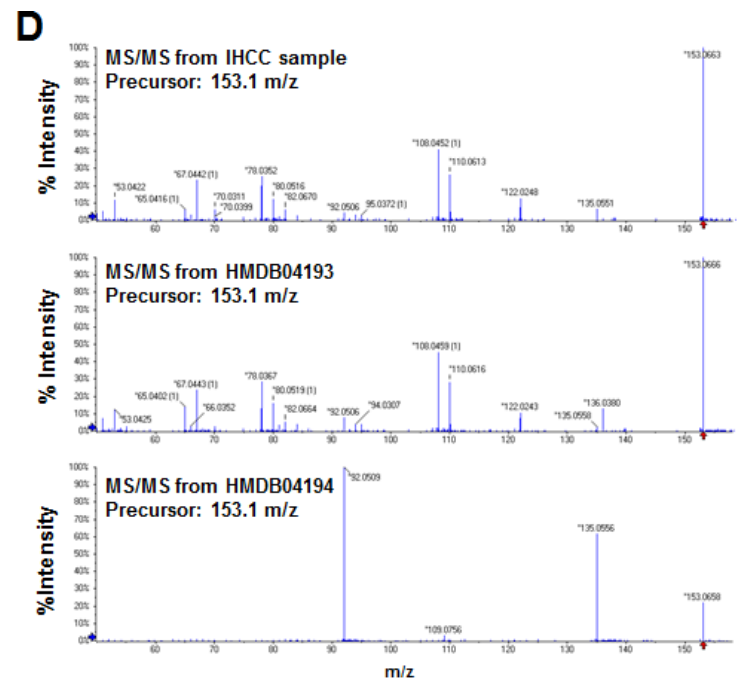

B

\begin{tabular}{|c|c|c|c|c|}
\hline \multicolumn{2}{|c|}{ Metabolic lon Information } & \multirow{2}{*}{ Formula } & $\begin{array}{c}\text { Mass Difference between metabolic ion } \\
\text { and candidate metabolite }(\mathrm{ppm})\end{array}$ & Candidate Metabolite in HMDB \\
\cline { 1 - 2 } $\mathrm{m} / \mathbf{z}$ & $\mathrm{RT}(\mathrm{min})$ & \multirow{2}{*}{ C7H8N2O2 } & -0.4 & $\begin{array}{r}\text { HMDB04193 } \\
\text { HMDB04194 }\end{array}$ \\
\hline
\end{tabular}

Figure 1: Identification of a metabolite ion of $\mathbf{m} / \mathbf{z} \mathbf{1 5 3 . 0 6 5 9}$ with an RT of $4.41 \mathbf{m i n}$. (A) The MS spectrum of the 153.0659 $\mathrm{m} / \mathrm{z}$ ion. The intensity of the ion was greater in serum from IHCC patients (red peaks) than control subjects (blue peaks). (B) Candidate HMDB metabolites giving rise to the $153.0659 \mathrm{~m} / \mathrm{z}$ ion. Based upon the MS information, the Formula Finder computational tools (SCIEX) selected two candidate metabolites as origins of the metabolic ion. (C) The structures of the candidate metabolites, HMDB04193 (2PY) and HMDB04194 (4PY). (D) MS/MS patterns at $153.0659 \mathrm{~m} / \mathrm{z}$ from IHCC sera, 2PY, and 4PY. The MS/MS IHCC pattern was identical to that of $2 \mathrm{PY}$. 
A

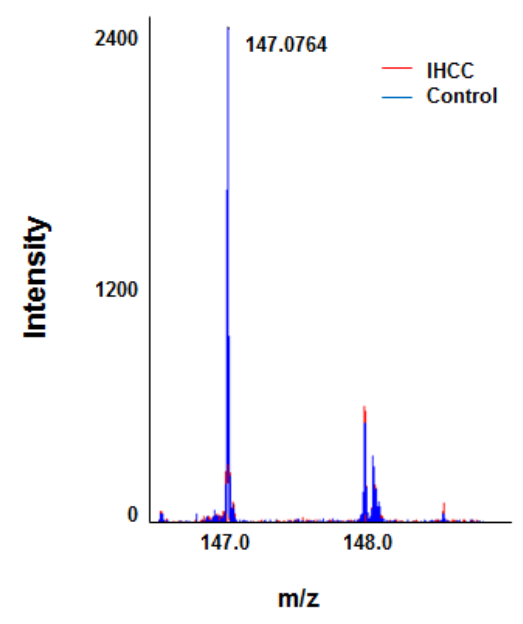

C<smiles>CCCCCCCCCCC(N)=O</smiles>

HMDB02031<smiles>CC(CNC(N)=O)C(=O)O</smiles>

D
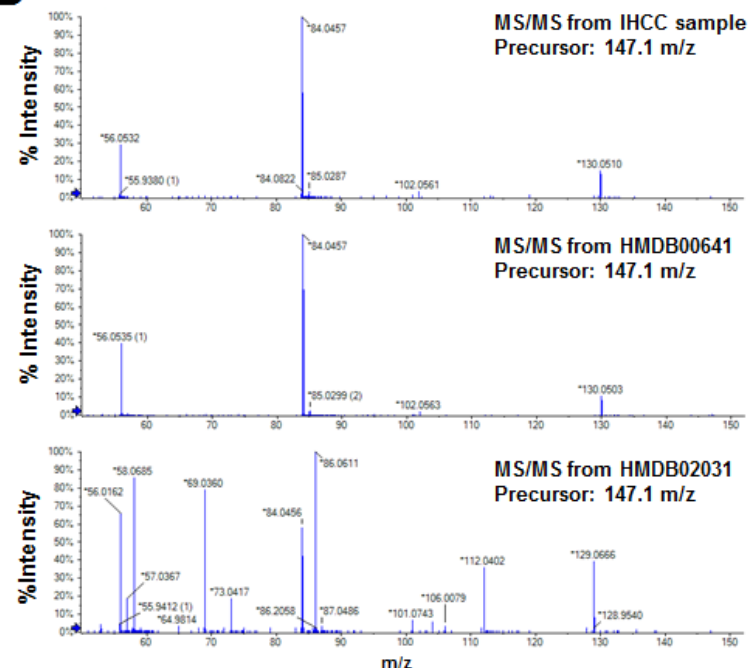

B

\begin{tabular}{|c|c|c|c|c|}
\hline \multicolumn{2}{|c|}{ Metabolic Ion Information } & \multirow{2}{*}{ Formula } & $\begin{array}{c}\text { Mass Difference between metabolic ion } \\
\text { and candidate metabolite }(\mathrm{ppm})\end{array}$ & Candidate Metabolite in HMDB \\
\cline { 1 - 2 } $\mathrm{m} / \mathbf{z}$ & $\mathrm{RT}(\mathrm{min})$ & & -0.1 & HMDB00641 \\
HMDB02031
\end{tabular}

Figure 2: Identification of a metabolic ion of $\mathbf{m} / \mathbf{z} \mathbf{1 4 7 . 0 7 6 4}$ with an RT of $1.44 \mathbf{m i n}$. (A) The MS spectrum at $147.0764 \mathrm{~m} / \mathrm{z}$. The intensity of the ion was lower in serum from IHCC patients (red peaks) than control subjects (blue peaks). (B) Candidate HMBD metabolites yielding $147.0764 \mathrm{~m} / \mathrm{z}$ ions. Two metabolic compounds were selected as candidates for the metabolic ion, HMDB00641 (L-glutamine) and HMDB02031 (ureidoisobutyric acid). (C) Structures of the candidate metabolites. (D) MS/MS patterns of the $147.0764 \mathrm{~m} / \mathrm{z}$ ions from L-glutamine and ureidoisobutyric acid. The MS/MS pattern of the IHCC metabolic ion of $147.0764 \mathrm{~m} / \mathrm{z}$ was identical to that of L-glutamine.

A

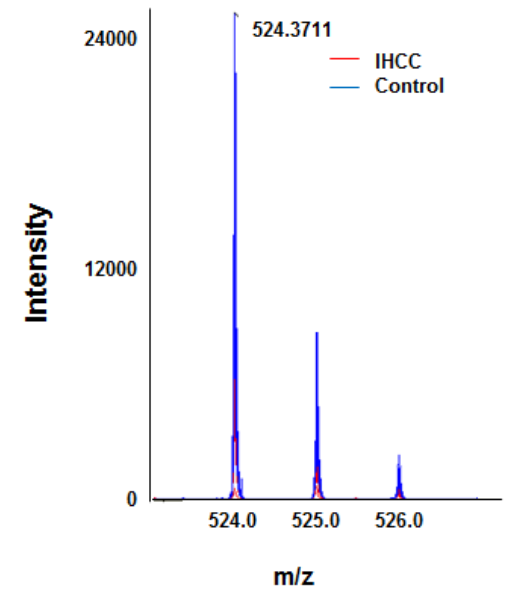

C

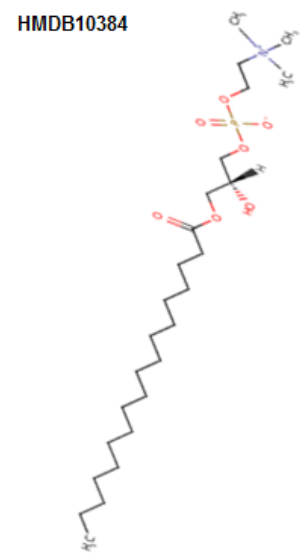

D

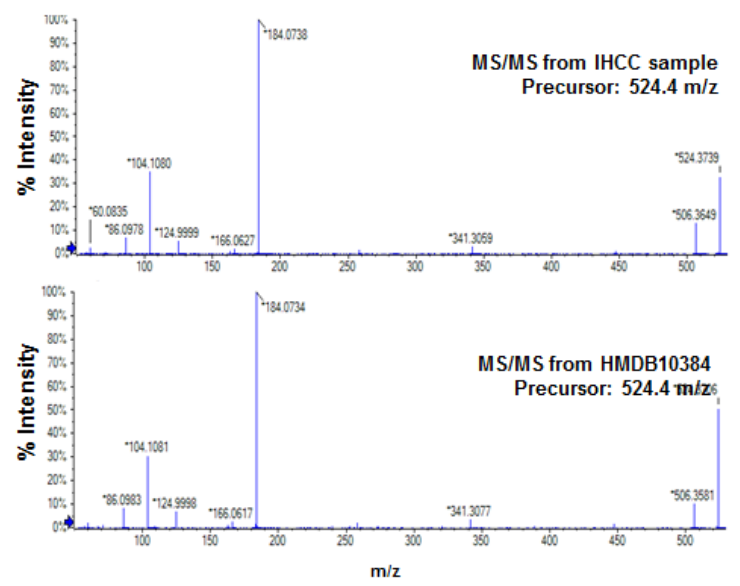

B

\begin{tabular}{|c|c|c|c|c|}
\hline \multicolumn{2}{|c|}{ Metabolic Ion Information } & \multirow{2}{*}{ Formula } & $\begin{array}{c}\text { Mass Difference between metabolic ion } \\
\text { and candidate metabolite }(\mathrm{ppm})\end{array}$ & Candidate Metabolite in HMDB \\
\cline { 1 - 2 } $\mathrm{m} / \mathbf{z}$ & $\mathrm{RT}(\mathrm{min})$ & 1.2 & HMDB10384 \\
\hline 524.3711 & 16.32 & C26H54NO7P & & 1.2 \\
\hline
\end{tabular}

Figure 3: Identification of a metabolic ion of $\mathbf{~ m / z ~} \mathbf{5 2 4 . 3 7 1 1}$ with an RT of $\mathbf{1 6 . 3 2} \mathbf{m i n}$. (A) The MS spectrum at $524.3711 \mathrm{~m} / \mathrm{z}$. The intensity of the ion was lower in serum from IHCC patients (red peaks) than control subjects (blue peaks). (B) A candidate HMDB metabolite giving rise to the $524.3711 \mathrm{~m} / \mathrm{z}$ ion. Only one metabolic candidate was identified: HMDB10384 (LPC 18:0). (C) Structure of the candidate metabolite. (D) MS/MS pattern of the $524.3711 \mathrm{~m} / \mathrm{z}$ ion from IHCC sera and LPC 18:0. The patterns were identical. 


\begin{tabular}{lcc}
\hline & Median & Cut-point \\
\hline L-glutamine (ng/ul) & 69.03 & 65.1 \\
2PY (pg/ul) & 520.36 & 587.05 \\
FPA (pg/ul) & 145.47 & 217.98 \\
Uric acid (ng/ul) & 28.17 & 31.92 \\
LPC16 (Arbitrary Unit) & 19214091.58 & 17966143.35 \\
LPC18 (Arbitrary Unit) & 928432.42 & 2230776.22 \\
\hline
\end{tabular}

N'-methyl-2-pyridone-5-carboxamide, 2PY; fibrinopeptide A, FPA; lysophosphatidylcholine, LPC.

coupled receptors. LPA is involved in many physiological and pathological processes, including cancer [25]. LPA enhances tumor growth, metastasis, and chemoresistance [26]. LPA is derived from lysophosphatidylcholine (LPC) via the action of a secreted phospholipase termed autotaxin (ATX), which was originally identified as an "autocrine motility factor" of tumor cells [24]. As ATX promotes both tumor formation and angiogenesis [24], and as ATX levels are elevated in several human malignancies [24, 27], many researchers believe that ATX may be a promising therapeutic target in patients with chronic inflammation and cancer $[25,26,28-30]$.

Similar to LPA and ATX, reduced LPC levels have been reported in the blood and tissue samples of patients with many types of cancer (serum from ovarian cancer patients [12]; and tissues from gastric [31], prostate [32], and liver [33] cancer patients). Higher LPC 18:0 plasma levels were associated with lower risks of breast, prostate, and colorectal cancers [34]. The plasma proportions of 18:1-LPC or 18:2-LPC (in terms of total saturated LPC levels), either individually or combined, are potential biomarkers for CRC [35]. Reduced LPC levels appear to be strongly associated with the activity of lysophosphatidylcholine acyltransferase 1 (LPCAT1), which converts LPC into phosphatidylcholine (PC). LPCAT1 was highly expressed in gastric cancer lesions compared to non-neoplastic mucosal tissues, predominantly in patients with differentiated adenocarcinomas [31]. PC and LPC 16:0 levels were higher and lower, respectively, in gastric cancer tissues of such patients [31]. LPCAT1 overexpression has been noted in many other types of cancer including breast [36]

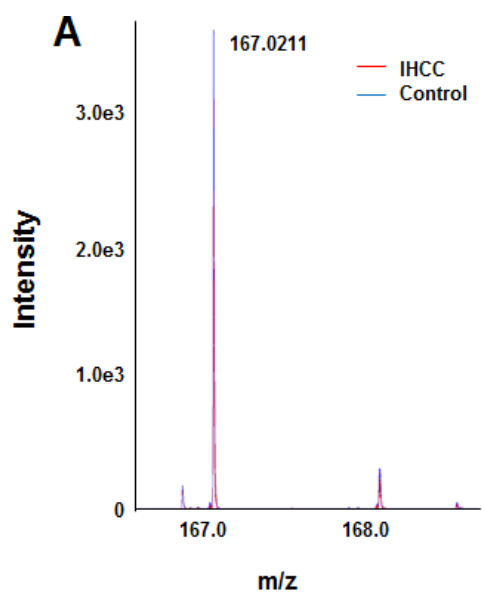

B

\begin{tabular}{|c|c|c|c|c|}
\hline \multicolumn{2}{|c|}{ Metabolic Ion Information } & \multirow{2}{*}{ Formula } & $\begin{array}{c}\text { Mass Difference between metabolic ion } \\
\text { and candidate metabolite }(\mathrm{ppm})\end{array}$ & Candidate Metabolite in HMDB \\
\cline { 1 - 2 } $\mathrm{m} / \mathbf{z}$ & $\mathrm{RT}(\mathrm{min})$ & & 0 & HMDB00289 \\
\hline 167.0211 & 2.48 & C5H4N4O3 & 0 \\
\hline
\end{tabular}

Figure 4: Identification of a metabolic ion of $\mathbf{m} / \mathbf{z} \mathbf{1 6 7 . 0 2 1 1} \mathbf{~ m} / \mathbf{z}$ with an RT of 2.48 min. (A) MS spectrum of the 167.0211 $\mathrm{m} / \mathrm{z}$ in the negative detection mode. The intensity of the ion was slightly less in serum from IHCC patients (red peaks) compared to controls (blue peaks). (B) The candidate HDMB metabolite giving rise to the ion of $167.0211 \mathrm{~m} / \mathrm{z}$. Only one candidate was identified: HMDB00289 (uric acid). (C) Structure of the candidate metabolite. (D) The MS/MS patterns of the $167.0211 \mathrm{~m} / \mathrm{z}$ from IHCC sera and uric acid. The patterns were identical. 
and colorectal [37] cancers, and the level has often been considered clinically relevant in tumorigenesis $[31,36$, 38-40].

Significantly lower levels of LPC were evident in the bile of patients with biliary tract cancer compared to those with benign biliary tract disease [41]. Therefore, LPC may be a novel biomarker allowing early detection of biliary tract cancer [30]. Recent studies have shown that LPC may trigger cholangiocyte senescence, thereby potentially contributing to the pathogenic development of biliary tract cancer [42]. LPC also inhibited cholangiocyte apoptosis by inducing COX-2 expression via a Raf-1dependent mechanism [43]. Such anti-apoptotic effects of LPC may play roles in biliary tract carcinogenesis in patients with anomalous pancreaticobiliary ductal junctions. Inhibition of these LPC actions may be a valuable chemopreventative strategy [43]. These studies support our finding that LPC 16:0 levels were reduced in the serum from IHCC patients, and strongly suggest that the LPC 16:0 levels may be metabolically significant in terms of IHCC prognosis.

We identified six metabolites (including 2PY and LPC 16:0) that were candidate metabolic biomarkers of IHCC. Of these, the 2PY and LPC 16:0 levels may be prognostic. Integration of information obtained upon quantification of the six individual metabolites identified in this study will lay a strong foundation for future validation of our results.

\section{MATERIALS AND METHODS}

\section{Chemicals and reagents}

LC-MS-grade acetonitrile and $0.1 \%(\mathrm{v} / \mathrm{v})$ formic acid (FA) in water were purchased from Thermo Scientific (Waltham, MA, USA). LC-MS-grade water was from Fisher Scientific (Hampton, NH, USA) and methanol was from Honeywell Burdick \& Jackson (Morris Plains, NJ, USA). L-glutamine, human FPA, uric acid, bovine serum albumin, and dichloromethane were obtained from Sigma-Aldrich (St. Louis, MO, USA). LPC 16:0 and LPC 18:0 were the products of Avanti Polar Lipids (Alabaster, Al, USA) and 2PY was obtained from Santa Cruz Biotechnology (Dallas, TX, USA). The internal standards were L-glutamine- $-{ }^{13} \mathrm{C}_{5}$, [Tyr ${ }^{\circ}$ ]-human FPA, uric acid-1,3- ${ }^{15} \mathrm{~N}_{2}$ (from Sigma-Aldrich) and 4PY (from Carbosynth [Compton, UK]). APCI-positive and ACPI-negative calibration solutions were the products of SCIEX (Framingham, MA, USA).
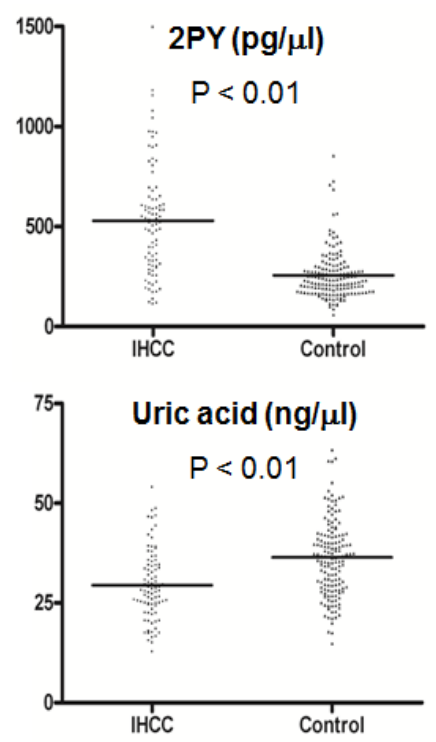
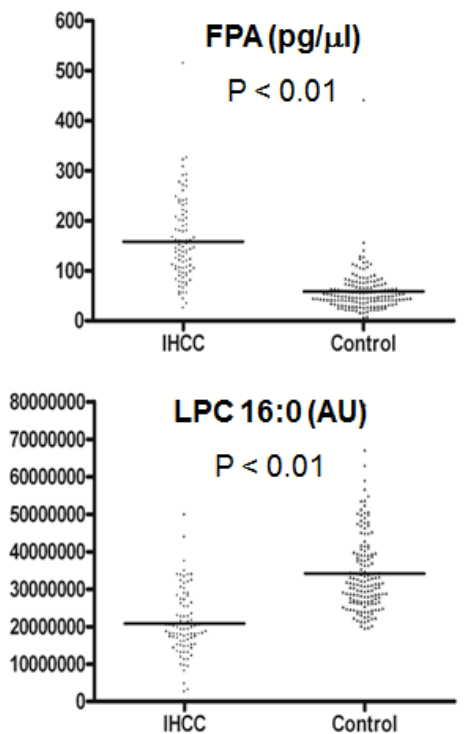
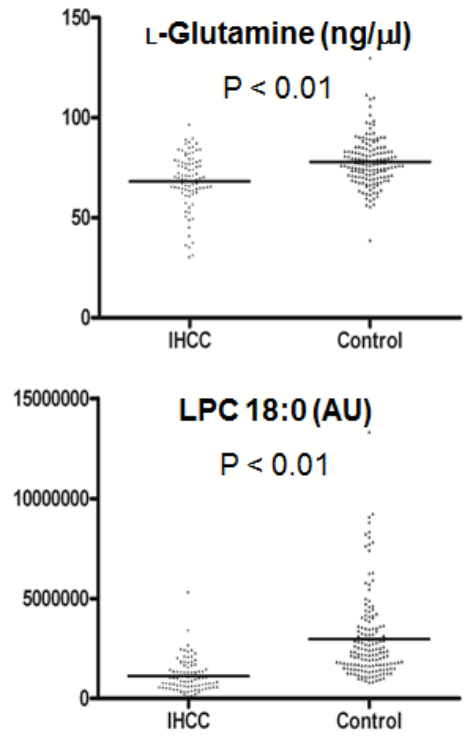

\begin{tabular}{|c|c|c|c|c|c|c|c|c|c|c|c|c|}
\hline \multirow{2}{*}{$\begin{array}{c}\text { IHCC }(n=87) \\
\text { Control }(n=150)\end{array}$} & \multicolumn{2}{|c|}{ 2PY (pg/pl) } & \multicolumn{2}{|c|}{ FPA (pg/ $/ \mu)$} & \multicolumn{2}{|c|}{ L-Glutamine $(\mathrm{ng} / \mu \mathrm{l})$} & \multicolumn{2}{|c|}{ Uric acid (ng/ll) } & \multicolumn{2}{|c|}{ LPC16:0 (AU) } & \multicolumn{2}{|c|}{ LPC18:0 (AU) } \\
\hline & IHCC & Control & IHCC & Control & IHCC & Control & IHCC & Control & IHCC & Control & IHCC & Control \\
\hline Min & 113.72 & 59.50 & 25.81 & 7.75 & 30.09 & 38.77 & 12.71 & 14.82 & 2619072.77 & 19615732.73 & 79639.20 & 812551.88 \\
\hline Max & 1495.29 & 854.74 & 515.00 & 442.10 & 96.25 & 129.93 & 53.95 & 70.88 & 49818664.34 & 79212517.63 & 5280681.24 & 13326355.42 \\
\hline Mean & 529.67 & 257.21 & 158.86 & 58.57 & 68.32 & 77.95 & 29.45 & 36.40 & 20818908.35 & 34163977.98 & 1115813.58 & 2972574.94 \\
\hline SD & 277.97 & 122.74 & 82.07 & 42.22 & 13.63 & 11.90 & 8.78 & 9.88 & 8595234.51 & 10387253.39 & 814923.58 & 2059398.56 \\
\hline
\end{tabular}

Figure 5: Quantification of six metabolites identified in sera of IHCC patients. Six metabolites (2PY, FPA, L-glutamine, uric acid, LPC 16:0, and LPC 18:0) that were differentially expressed in sera from IHCC patients (compared to controls) were quantified using the MRM MS mode. 2PY and FPA levels were increased ca. 2- and 2.5-fold in sera from IHCC patients compared to controls, respectively (both $p<0.01$ ). By contrast, L-glutamine, uric acid, LPC 16:0, and LPC 18:0 levels were reduced in sera from IHCC patients (all $p<$ 0.01). AU: arbitrary unit. 


\section{A $2 \mathrm{PY}(\mathrm{pg} / \mu \mathrm{l})$}
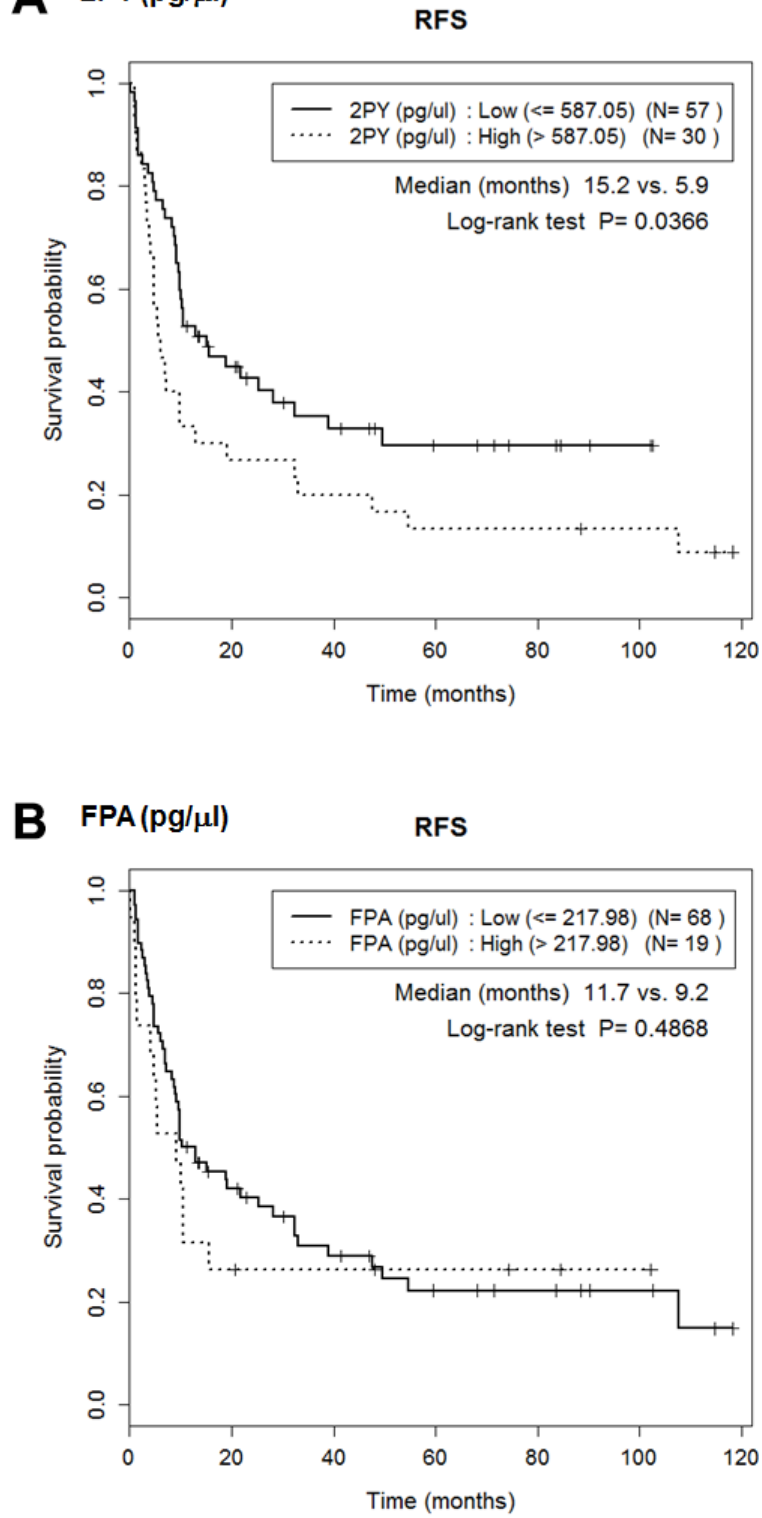

C L-Glutamine (ng/ $/ \mathrm{ll})$







os

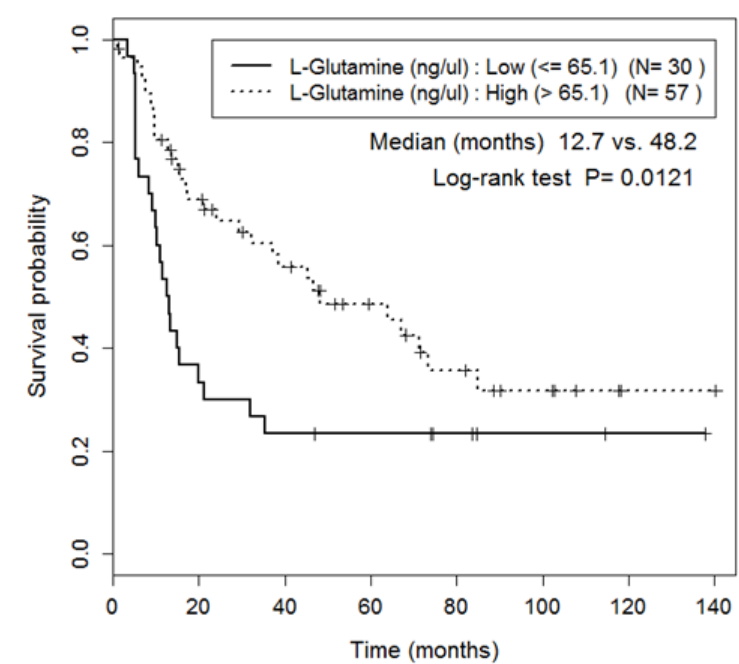


D Uric acid (ng/ul)



E LPC 16:0(AU)

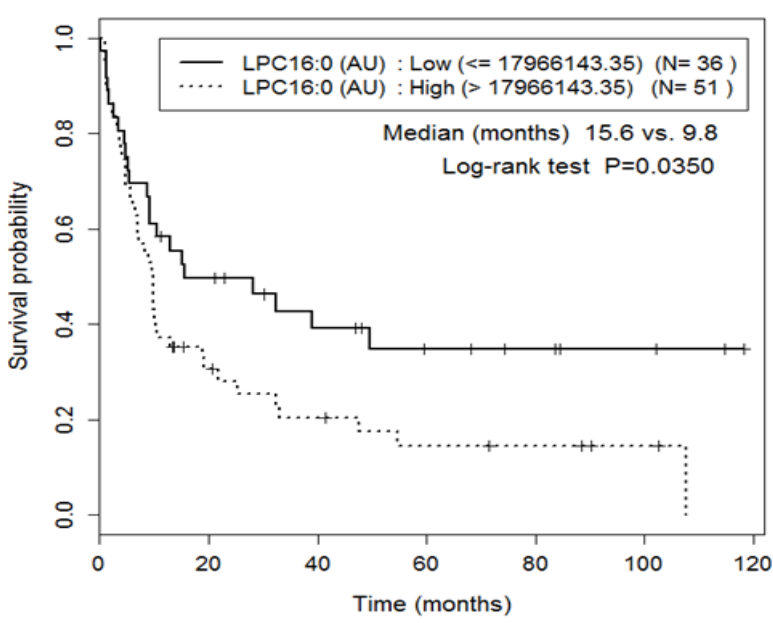

F LPC 18:0(AU)

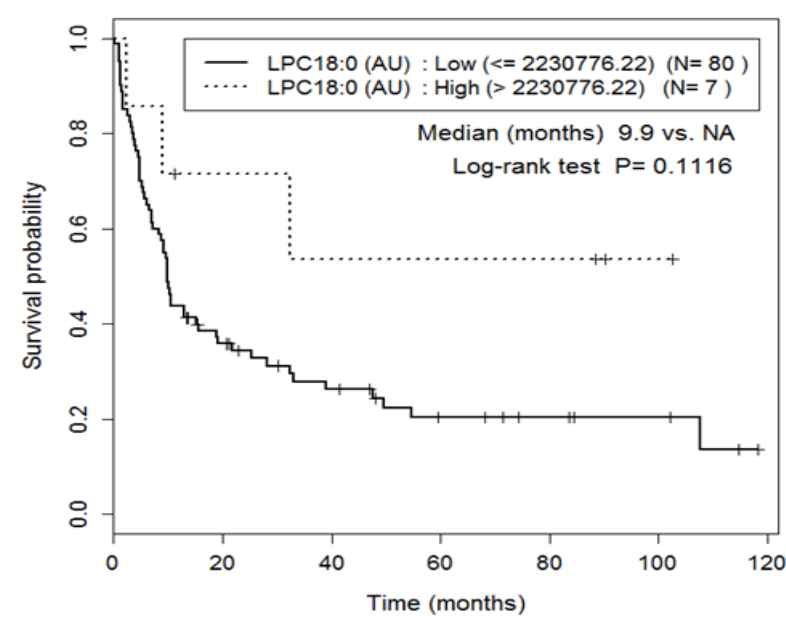

OS



os

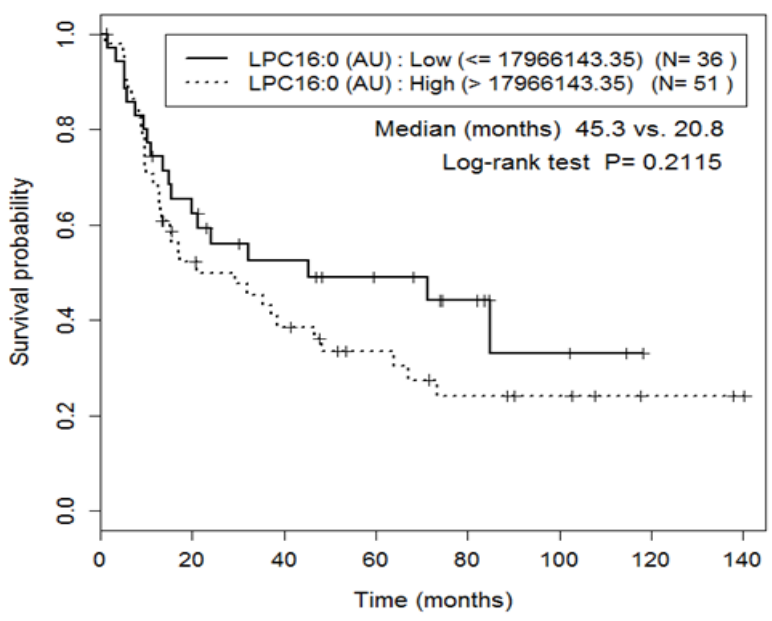

os

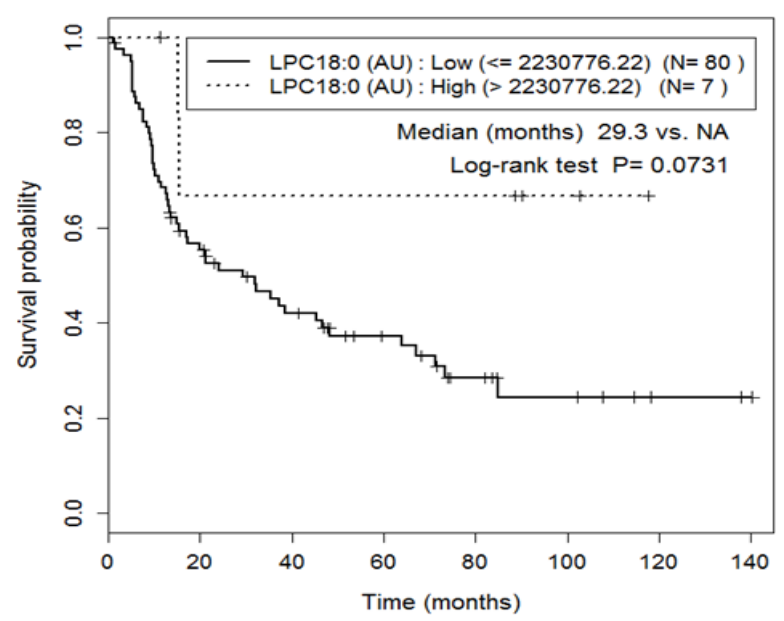

Figure 6: Lower 2PY and LPC16 levels were associated with better RFS. Cutoffs for maximal prediction of high/low level RFSs were determined (Table 3). The Figure shows the associations between RFS or overall survival (OS) with the levels of the six metabolites, 2PY (A), FPA(B), L-glutamine (C), uric acid (D), LPC 16:0 (E) and LPC 18:0 (F). 2PY and LPC 16:0 showed significantly better RFS at low level than high level ( $p=0.037$ and $p=0.035$, respectively). In addition, high-level L-glutamine was associated with a better OS $(p=0.0121)$. 


\section{Serum extraction for metabolite profiling}

A total of 237 serum samples from IHCC patients $(n=87)$ and healthy controls $(n=150)$ were extracted using the modified Bligh and Dyer method (14). In brief, $50 \mu \mathrm{L}$ serum was added to $1 \mathrm{~mL}$ water. After vortexing, 2 $\mathrm{mL} \mathrm{MeOH}$ and $0.9 \mathrm{~mL}$ dichloromethane were added. After vortexing and placing on ice for $30 \mathrm{~min}, 1 \mathrm{~mL}$ water and $0.9 \mathrm{~mL}$ dichloromethane were added, and the mixture was centrifuged (1,500 rpm, $10 \mathrm{~min}$, room temperature). The supernatant was dried under an $\mathrm{N}_{2}$ stream and subjected to MS analysis.

\section{Serum metabolite profiling}

The dried samples were reconstituted in $0.1 \%(\mathrm{v} / \mathrm{v})$ FA and subjected to liquid chromatography (LC)-MS/MS using a Shimadzu Nexera X2 system (Shimadzu, Chiyodaku, Tokyo, Japan) coupled to a SCIEX Triple TOF 5600+ module (SCIEX) equipped at the front end with a DuoSpray ion source. For ultra-high-performance LC, samples were loaded into Atlantis T3 sentry guard cartridges ( $3 \mu \mathrm{m}, 2.1$ $\mathrm{mm} \times 10 \mathrm{~mm}$; Waters Corporation [Milford, MA, USA]) and separation proceeded on an Atlantis T3 column ( $3 \mu \mathrm{m}$, $2.1 \mathrm{~mm} \times 100 \mathrm{~mm}$; Waters). The MS system was set to perform one full scan ( 50 to $1,200 \mathrm{~m} / \mathrm{z}$ ) followed by MS/ MS of the 10 most abundant parent ions (mass tolerance, 50 $\mathrm{mDa}$; collision energy, 35\%).

\section{Selection and identification of metabolite ions}

LC/MS peak lists (.peaks files) were created for all of the samples (.wiff files) using MarkerView software running under the following constraints: minimum retention time, $0.00 \mathrm{~min}$; subtraction offset, 10 scans; subtraction multiplication factor, 1.3; noise threshold, 10; minimum spectral peak width, $10 \mathrm{ppm}$; and minimum RT peak width, 5 scans. Next, a peak table was created by simultaneously importing the LC/MS peak lists for all of the samples. The parameters for the second process were as follows: RT tolerance, $0.01 \mathrm{~min}$; mass tolerance, 10.0 ppm; intensity threshold, 10; and maximum number of peaks, 20,000. Areas were derived using raw data, and not using the original peak findings, as suggested by the reference manual. The resulting peak table was normalized using the "total area sums" method and the data converted into common logarithms. The MS and MS/MS spectra were submitted to the Formula Finder computational tools (SCIEX) that propose probable elemental compositions within a specified mass tolerance of a given mass-tocharge ratio $(\mathrm{m} / \mathrm{z})$, using PeakView software (SCIEX). By interrogating the HMDB metabolite databases, the specific compounds giving rise to the observed $\mathrm{m} / \mathrm{z}$ ions were identified, and listed in rank order based on the MS and MS/MS data. Proteomic MS/MS analyses were performed with the aid of ProteinPilot software (SCIEX).

\section{Quantification of the six selected metabolites}

For quantitative analyses, serum samples were mixed with L-glutamine- ${ }^{13} \mathrm{C}_{5}$, [Tyr]-human FPA, uric acid-1,3- ${ }^{15} \mathrm{~N}_{2}$, LPC 16:0-d31, and 4PY (internal standards). Varying amounts of standards were spiked into bovine serum albumin solutions in the presence of the internal standard, and extracted. MS analyses were performed using a Triple TOF $5600+$ system (SCIEX) fitted with an analyst data acquisition module. The positive ion multiple reaction monitoring (MRM) mode was used for quantitative analysis of L-glutamine, LPC 16:0, LPC 18:0, 2PY, and FPA. Sample extracts $(5 \mu \mathrm{L})$ were loaded via a Nexera X2 LC system (Shimadzu) onto a three-step linear gradient (solvent A, $0.1 \%[\mathrm{v} / \mathrm{v}]$ FA in water; solvent B, $100 \%$ ACN; $1 \%$ solvent $\mathrm{B}$ for $1.5 \mathrm{~min}, 1-25 \%$ B for $4.5 \mathrm{~min}, 25-45 \%$ B for $2 \mathrm{~min}$, $45-90 \%$ B for $2 \mathrm{~min}, 90 \%$ B for $4 \mathrm{~min}, 90-1 \%$ B for $0.5 \mathrm{~min}$, and $1 \% \mathrm{~B}$ for $5.5 \mathrm{~min}$ ). The negative ion MRM mode was used for quantitative analysis of uric acid levels. LC separations (10 min/sample) were performed using a one-step linear gradient (solvent A, $0.1 \%[\mathrm{v} / \mathrm{v}]$ FA in water; solvent $\mathrm{B}, 100 \% \mathrm{ACN} ; 1 \%$ solvent $\mathrm{B}$ for $1.5 \mathrm{~min}, 1-90 \%$ B for $2.5 \mathrm{~min}, 90 \%$ B for $2 \mathrm{~min}$, $90-1 \%$ B for $0.5 \mathrm{~min}$, and $1 \%$ B for $3.5 \mathrm{~min}$ ). The six metabolites were quantified with the aid of MultiQuant software (SCIEX).

\section{Statistical analysis}

The Cox proportional hazard model was used to test for difference in RFS between two groups dichotomized according to CA 19-9 level. The levels of continuous biomarkers were dichotomized based on cutoffs maximizing the differences between groups in terms of the log-rank test statistic [44]. The RFS differences between the two groups were compared using the log-rank test, and survival was estimated by drawing Kaplan-Meier curves.

\section{ACKNOWLEDGMENTS AND FUNDING}

This work was supported by the grants from the Basic Science Research Program through the National Research Foundation of Korea (NRF) funded by the Ministry of Science and ICT (NRF-2017R1A2B4007859), the Industry Technology Development Program (10063335, Development of Nanotechnology-based Quantitative Surface Mass Spectrometric Platform Technique to Detect Low-Mass Biomolecules) funded by the Ministry of Trade, Industry \& Energy (MOTIE), and the National Cancer Center (Grant number 1610040-2), Republic of Korea.

\section{CONFLICTS OF INTEREST}

The authors declare no conflicts of interest. 


\section{REFERENCES}

1. de Groen PC, Gores GJ, LaRusso NF, Gunderson LL, Nagorney DM. Biliary tract cancers. N Engl J Med. 1999; 341:1368-78. https://doi.org/10.1056/NEJM199910283411807.

2. Shaib Y, El-Serag HB. The epidemiology of cholangiocarcinoma. Semin Liver Dis. 2004; 24:115-25. https://doi.org/10.1055/s-2004-828889.

3. Shin HR, Jung KW, Won YJ, Park JG, and 139 KCCRaffiliated Hospitals. 2002 annual report of the Korea Central Cancer Registry: based on registered data from 139 hospitals. Cancer Res Treat. 2004; 36:103-14. https://doi. org/10.4143/crt.2004.36.2.103.

4. Patel T. Worldwide trends in mortality from biliary tract malignancies. BMC Cancer. 2002; 2:10. https://doi. org/10.1186/1471-2407-2-10.

5. Koprowski H, Steplewski Z, Mitchell K, Herlyn M, Herlyn D, Fuhrer P. Colorectal carcinoma antigens detected by hybridoma antibodies. Somatic Cell Genet. 1979; 5:957-71. https://doi.org/10.1007/BF01542654.

6. Patel AH, Harnois DM, Klee GG, LaRusso NF, Gores GJ. The utility of CA 19-9 in the diagnoses of cholangiocarcinoma in patients without primary sclerosing cholangitis. Am J Gastroenterol. 2000; 95:204-07. https:// doi.org/10.1111/j.1572-0241.2000.01685.x.

7. Tao LY, Cai L, He XD, Liu W, Qu Q. Comparison of serum tumor markers for intrahepatic cholangiocarcinoma and hepatocellular carcinoma. Am Surg. 2010; 76:1210-13.

8. Uenishi T, Kubo S, Hirohashi K, Tanaka H, Shuto T, Yamamoto T, Nishiguchi S. Cytokeratin-19 fragments in serum (CYFRA 21-1) as a marker in primary liver cancer. Br J Cancer. 2003; 88:1894-99. https://doi.org/10.1038/sj.bjc.6601026.

9. Lee JH, Yoo BC, Kim YH, Ahn SA, Yeo SG, Cho JY, Kim $\mathrm{KH}$, Kim SC. Low-mass-ion discriminant equation (LOME) for ovarian cancer screening. BioData Min. 2016; 9:32. https://doi.org/10.1186/s13040-016-0111-7.

10. Roh K, Yeo SG, Yoo BC, Kim KH, Kim SY, Kim MJ. Seven low-mass ions in pretreatment serum as potential predictive markers of the chemoradiotherapy response of rectal cancer. Anticancer Drugs. 2016; 27:787-93. https:// doi.org/10.1097/CAD.0000000000000391.

11. Kim K, Yeo SG, Yoo BC. Identification of hypoxanthine and phosphoenolpyruvic Acid as serum markers of chemoradiotherapy response in locally advanced rectal cancer. Cancer Res Treat. 2015; 47:78-89. https://doi. org/10.4143/crt.2013.127.

12. Kim SC, Kim MK, Kim YH, Ahn SA, Kim KH, Kim K, Kim WK, Lee JH, Cho JY, Yoo BC. Differential levels of L-homocysteic acid and lysophosphatidylcholine (16:0) in sera of patients with ovarian cancer. Oncol Lett. 2014; 8:566-74.

13. Lee JH, Kim SC, Kim KH, Kim WK, Lee MJ, Yoo BC, Yeo SG. Decreased cellular levels of palmitic amide are linked to 5-fluorouracil resistance in human colon cancer cells. Hepatogastroenterology. 2014; 61:343-48.
14. Lee JH, Kim KH, Park JW, Chang HJ, Kim BC, Kim SY, Kim KG, Lee ES, Kim DY, Oh JH, Yoo BC, Kim IH. Low-mass-ion discriminant equation: a new concept for colorectal cancer screening. Int J Cancer. 2014; 134:184453. https://doi.org/10.1002/ijc.28517.

15. Yoo BC, Kong SY, Jang SG, Kim KH, Ahn SA, Park WS, Park S, Yun T, Eom HS. Identification of hypoxanthine as a urine marker for non-Hodgkin lymphoma by lowmass-ion profiling. BMC Cancer. 2010; 10:55. https://doi. org/10.1186/1471-2407-10-55.

16. Slominska EM, Smolenski RT, Szolkiewicz M, Leaver N, Rutkowski B, Simmonds HA, Swierczynski J. Accumulation of plasma N-methyl-2-pyridone-5-carboxamide in patients with chronic renal failure. Mol Cell Biochem. 2002; 231:83-88. https://doi.org/10.1023/A:1014445329756.

17. Rutkowski B, Slominska E, Szolkiewicz M, Smolenski RT, Striley C, Rutkowski P, Swierczynski J. N-methyl-2-pyridone5-carboxamide: a novel uremic toxin? Kidney Int Suppl. 2003; 84:S19-21. https://doi.org/10.1046/j.1523-1755.63.s84.36.x.

18. Slominska EM, Kowalik K, Smolenski RT, Szolkiewicz M, Rutkowski P, Rutkowski B, Swierczynski J. Accumulation of poly(ADP-ribose) polymerase inhibitors in children with chronic renal failure. Pediatr Nephrol. 2006; 21:800-06. https://doi.org/10.1007/s00467-006-0072-z.

19. Carrey EA, Smolenski RT, Edbury SM, Laurence A, Marinaki AM, Duley JA, Zhu LM, Goldsmith DJ, Simmonds HA. An unusual pyridine nucleotide accumulating in erythrocytes: its identity and positive correlation with degree of renal failure. Nucleosides Nucleotides Nucleic Acids. 2004; 23:1135-39. https://doi. org/10.1081/NCN-200027391.

20. Carrey EA, Smolenski RT, Edbury SM, Laurence A, Marinaki AM, Duley JA, Zhu L, Goldsmith DJ, Simmonds HA. Origin and characteristics of an unusual pyridine nucleotide accumulating in erythrocytes: positive correlation with degree of renal failure. Clin Chim Acta. 2003; 335:11729. https://doi.org/10.1016/S0009-8981(03)00294-8.

21. Rutkowski P, Słominska EM, Szołkiewicz M, Aleksandrowicz E, Smolenski RT, Wołyniec W, Renke M, Wisterowicz K, Swierczynski J, Rutkowski B. Relationship between uremic toxins and oxidative stress in patients with chronic renal failure. Scand J Urol Nephrol. 2007; 41:24348. https://doi.org/10.1080/00365590601017170.

22. Slominska EM, Smolenski RT, Osborne F, Swierczynski J, Yacoub MH. The effect of N-methyl-2-pyridone-5carboxamide - A nicotinamide catabolite on poly ADPrybosylation and oxidative stress injury in endothelial cells. Nucleosides Nucleotides Nucleic Acids. 2005; 24:259-62.

23. Pumpo R, Sarnelli G, Spinella A, Budillon G, Cuomo R. The metabolism of nicotinamide in human liver cirrhosis: a study on N-methylnicotinamide and 2-pyridone-5-carboxamide production. Am J Gastroenterol. 2001; 96:1183-87. https:// doi.org/10.1111/j.1572-0241.2001.03698.x.

24. Houben AJ, Moolenaar WH. Autotaxin and LPA receptor signaling in cancer. Cancer Metastasis Rev. 2011; 30:55765. https://doi.org/10.1007/s10555-011-9319-7. 
25. Volden PA, Skor MN, Johnson MB, Singh P, Patel FN, McClintock MK, Brady MJ, Conzen SD. Mammary Adipose Tissue-Derived Lysophospholipids Promote Estrogen Receptor-Negative Mammary Epithelial Cell Proliferation. Cancer Prev Res (Phila). 2016; 9:367-78. https://doi.org/10.1158/1940-6207.CAPR-15-0107.

26. Benesch MG, Ko YM, Tang X, Dewald J, LopezCampistrous A, Zhao YY, Lai R, Curtis JM, Brindley DN, McMullen TP. Autotaxin is an inflammatory mediator and therapeutic target in thyroid cancer. Endocr Relat Cancer. 2015; 22:593-607. https://doi.org/10.1530/ERC-15-0045.

27. Nakamura K, Igarashi K, Ohkawa R, Yokota H, Masuda A, Nakagawa S, Yano T, Ikeda H, Aoki J, Yatomi Y. Serum autotaxin is not a useful biomarker for ovarian cancer. Lipids. 2012; 47:927-30. https://doi.org/10.1007/s11745012-3691-0.

28. Barbayianni E, Magrioti V, Moutevelis-Minakakis P, Kokotos G. Autotaxin inhibitors: a patent review. Expert Opin Ther Pat. 2013; 23:1123-1132. https://doi.org/10.151 7/13543776.2013.796364.

29. Teo K, Brunton VG. The role and therapeutic potential of the autotaxin-lysophosphatidate signalling axis in breast cancer. Biochem J. 2014; 463:157-65. https://doi. org/10.1042/BJ20140680.

30. Barbayianni E, Kaffe E, Aidinis V, Kokotos G. Autotaxin, a secreted lysophospholipase D, as a promising therapeutic target in chronic inflammation and cancer. Prog Lipid Res. 2015; 58:76-96. https://doi.org/10.1016/j.plipres.2015.02.001.

31. Uehara T, Kikuchi H, Miyazaki S, Iino I, Setoguchi T, Hiramatsu Y, Ohta M, Kamiya K, Morita Y, Tanaka H, Baba S, Hayasaka T, Setou M, Konno H. Overexpression of Lysophosphatidylcholine Acyltransferase 1 and Concomitant Lipid Alterations in Gastric Cancer. Ann Surg Oncol. 2016 (Suppl 2); 23:S206-13. https://doi. org/10.1245/s10434-015-4459-6.

32. Goto T, Terada N, Inoue T, Kobayashi T, Nakayama K, Okada Y, Yoshikawa T, Miyazaki Y, Uegaki M, Utsunomiya N, Makino Y, Sumiyoshi S, Yamasaki T, et al. Decreased expression of lysophosphatidylcholine $(16: 0 / \mathrm{OH})$ in high resolution imaging mass spectrometry independently predicts biochemical recurrence after surgical treatment for prostate cancer. Prostate. 2015; 75:1821-30. https://doi. org/10.1002/pros.23088.

33. Morita Y, Sakaguchi T, Ikegami K, Goto-Inoue N, Hayasaka T, Hang VT, Tanaka H, Harada T, Shibasaki Y, Suzuki A, Fukumoto K, Inaba K, Murakami M, et al. Lysophosphatidylcholine acyltransferase 1 altered phospholipid composition and regulated hepatoma progression. J Hepatol. 2013; 59:292-99. https://doi. org/10.1016/j.jhep.2013.02.030.

34. Kühn T, Floegel A, Sookthai D, Johnson T, RolleKampczyk U, Otto W, von Bergen M, Boeing H, Kaaks R. Higher plasma levels of lysophosphatidylcholine 18:0 are related to a lower risk of common cancers in a prospective metabolomics study. BMC Med. 2016; 14:13. https://doi. org/10.1186/s12916-016-0552-3.
35. Zhao Z, Xiao Y, Elson P, Tan H, Plummer SJ, Berk M, Aung PP, Lavery IC, Achkar JP, Li L, Casey G, Xu Y. Plasma lysophosphatidylcholine levels: potential biomarkers for colorectal cancer. J Clin Oncol. 2007; 25:2696-701. https:// doi.org/10.1200/JCO.2006.08.5571.

36. Abdelzaher E, Mostafa MF. Lysophosphatidylcholine acyltransferase 1 (LPCAT1) upregulation in breast carcinoma contributes to tumor progression and predicts early tumor recurrence. Tumour Biol. 2015; 36:5473-83. https://doi.org/10.1007/s13277-015-3214-8.

37. Mansilla F, da Costa KA, Wang S, Kruhøffer M, Lewin TM, Orntoft TF, Coleman RA, Birkenkamp-Demtröder K. Lysophosphatidylcholine acyltransferase 1 (LPCAT1) overexpression in human colorectal cancer. J Mol Med (Berl). 2009; 87:85-97. https://doi.org/10.1007/s00109-008-0409-0.

38. Faas FH, Dang AQ, White J, Schaefer R, Johnson D. Increased prostatic lysophosphatidylcholine acyltransferase activity in human prostate cancer: a marker for malignancy. $\mathrm{J}$ Urol. 2001; 165:463-68. https://doi.org/10.1097/00005392200102000-00026.

39. Grupp K, Sanader S, Sirma H, Simon R, Koop C, Prien K, Hube-Magg C, Salomon G, Graefen M, Heinzer H, Minner S, Izbicki JR, Sauter G, et al. High lysophosphatidylcholine acyltransferase 1 expression independently predicts high risk for biochemical recurrence in prostate cancers. Mol Oncol. 2013; 7:1001-11. https://doi.org/10.1016/j.molonc.2013.07.009.

40. Shida-Sakazume T, Endo-Sakamoto $Y$, Unozawa $M$, Fukumoto C, Shimada K, Kasamatsu A, Ogawara K, Yokoe H, Shiiba M, Tanzawa H, Uzawa K. Lysophosphatidylcholine acyltransferase 1 overexpression promotes oral squamous cell carcinoma progression via enhanced biosynthesis of plateletactivating factor. PLoS One. 2015; 10:e120143. https://doi. org/10.1371/journal.pone.0120143.

41. Xu X, Cheng S, Ding C, Lv Z, Chen D, Wu J, Zheng S. Identification of bile biomarkers of biliary tract cancer through a liquid chromatography/mass spectrometry-based metabolomic method. Mol Med Rep. 2015; 11:2191-98. https://doi.org/10.3892/mmr.2014.2973.

42. Shimizu R, Kanno K, Sugiyama A, Ohata H, Araki A, Kishikawa N, Kimura Y, Yamamoto H, Kodama M, Kihira K, Tazuma S. Cholangiocyte senescence caused by lysophosphatidylcholine as a potential implication in carcinogenesis. J Hepatobiliary Pancreat Sci. 2015; 22:67582. https://doi.org/10.1002/jhbp.256.

43. Gwak GY, Yoon JH, Lee SH, Lee SM, Lee HS, Gores GJ. Lysophosphatidylcholine suppresses apoptotic cell death by inducing cyclooxygenase-2 expression via a Raf-1 dependent mechanism in human cholangiocytes. J Cancer Res Clin Oncol. 2006; 132:771-79. https://doi.org/10.1007/ s00432-006-0125-5.

44. Contal C, O'Quigley J. An application of change point methods in studying the effect of age on survival in breast cancer. Comput Stat Data Anal. 1999; 30:253-70. https:// doi.org/10.1016/S0167-9473(98)00096-6. 